\title{
Formation of calcium pyrophosphate crystals in vitro: implications for calcium pyrophosphate crystal deposition disease (pseudogout)
}

\author{
P. R. HEARN AND R. G. G. RUSSELL
}

From the Department of Human Metabolism and Clinical Biochemistry, University of Sheffield Medical School, Beech Hill Road, Sheffield S10 2RX

SUMMARY Little is known about how calcium pyrophosphate dihydrate (CaPPD) crystals form in vivo and give rise to chondrocalcinosis or pseudogout (pyrophosphate arthropathy or calcium pyrophosphate crystal deposition disease). In this study a simple method has been devised to define the conditions necessary for the deposition of crystals in vitro. Crystal formation is monitored by ${ }^{45} \mathrm{Ca}$ in the presence of $1.5 \mathrm{mmol} / 1 \mathrm{Ca}$ and increasing concentrations of inorganic pyrophosphate (PPi) under simulated physiological conditions of $\mathrm{pH}$ and ionic strength. Concentrations of PPi required to initiate crystal formation were about $40 \mathrm{mmol} / 1$ in the absence and $175 \mathrm{mmol} / 1$ in the presence of $0.5 \mathrm{mmol} / 1 \mathrm{Mg}^{2+}$ at $\mathrm{pH}$ 7.4. Less $\mathrm{PPi}$ was required at higher $\mathrm{pH}$ values. The naturally occurring monoclinic and triclinic forms of CaPPD were produced after prolonged incubation in vitro, but the initial deposits were amorphous or orthorhombic. The physiological significance of these observations is discussed. Since much higher concentrations of PPi are required to form crystals in vitro than are found to occur naturally in synovial fluids from patients with pyrophosphate arthropathy, it is suggested that crystals are more likely to deposit initially within cartilage and that nucleating mechanisms may be important in vivo. Since other workers have observed a slow interconversion of other calcium pyrophosphate crystal forms into monoclinic and triclinic allomorphs under laboratory conditions, the reason why only these 2 forms occur under clinical conditions may reflect the long time available in vivo for the formation of crystals.

Calcium pyrophosphate dihydrate (CaPPD) crystals were first identified in articular cartilage and synovial fluid by McCarty et al. (1962). Their studies firmly established the existence of a new clinical disorder, previously confused with gout, for which they proposed the name pseudogout. Subsequently the main features of this clinical syndrome have been well documented (Bundens et al., 1965; McCarty, 1976), and the condition is now known as calcium pyrophosphate crystal deposition disease.

The concentrations of inorganic pyrophosphate (PPi) in synovial fluids from patients with pseudogout (PG) are higher than in control fluids (Russell et al., 1970). Higher levels of PPi also occur in joint fluids from patients with osteoarthritis (OA) or urate gout than in patients with rheumatoid arthritis or traumatic joint effusions (McCarty et al., Accepted for publication 15 May 1979

Correspondence to Dr Hearn.
1971; Altman et al., 1973). Silcox and McCarty (1974) in a more extensive series found mean levels of PPi to be similar in 35 osteoarthritic and 29 pseudogout synovial fluids. They suggested that the raised levels were in both cases possibly attributable to increased PPi production as a result of enhanced biosynthesis of macromolecules by articular cartilage.

It is unknown to what extent the solubilisation of CaPPD crystals contributes to synovial PPi levels. However, because of the slow rate of dissolution of crystals compared with the turnover time of the PPi pool (Camerlain et al., 1975), it is probable that PPi levels in synovial fluid are determined more by the balance between rates of synthesis and removal of PPi from the joint than by the presence or absence of crystals. Indeed it would appear that synovial fluid PPi levels alone are probably not the major determinant for CaPPD crystal 
formation, because although OA and PG fluids show similar PPi levels in one series (Silcox and McCarty, 1974) only the PG group had significant numbers of CaPPD crystals in their fluids.

There has been some speculation about the physical mechanisms by which CaPPD crystals come to be shed into joint fluids (McCarty, 1976). It is suggested that crystals probably form in the articular cartilage, perhaps around chondrocytes, and are then released from pockets (lacunae) as the overlying cartilage layers are slowly eroded. However, no studies have been reported in which the artificial growth of these crystals has been simulated in vitro.

The present study sets out to define the conditions necessary for CaPPD crystal growth and also to investigate some of the mechanisms which may be involved in forming the clinically observed crystals in vivo.

\section{Materials and methods}

All chemicals were of Analar grade.

Crystal growth was initiated in solutions the compositions of which was designed to resemble physiological fluids. Since the purpose of the study was to test the effect of addition of various solutes on crystal growth we decided not to start with a multisolute buffer such as Ringer's solution. The composition of the buffer used is shown in Table 1 .

The final solute concentration was adjusted to $310 \mathrm{mmol} / \mathrm{l}$ by varying the amount of $\mathrm{NaCl}$ in each case. The $\mathrm{pH}$ was $7 \cdot 4$ unless otherwise indicated.
Table 1 Buffer for crystal growth studies

\begin{tabular}{lll}
\hline & \multicolumn{2}{c}{ Concentrations (mmol) } \\
\cline { 2 - 3 } & In buffer & In synovial fluid \\
\hline Tris & 40 & - \\
$\mathrm{Na}$ & 112 & 136 \\
$\mathrm{~K}$ & 4 & 4 \\
$\mathrm{Mg}$ & $0 \cdot 5-1 \cdot 0$ & $0 \cdot 5-1 \cdot 0$ \\
$\mathrm{Ca}$ & 1.5 & 1.5 \\
$\mathrm{Cl}$ & 150 & 150 \\
$\mathrm{PPi}$ & Various & 0.004 \\
\hline
\end{tabular}

*Source: Documenta Geigy. Values are approximate.

All individual solutions were made with fresh deionised water and were filtered through $0.45 \mu \mathrm{m}$ pore size Millipore filters (HAWPO 1300) to remove fine particles immediately before use. Glassware was thoroughly rinsed with deionised water before use, and vessels were covered at all times in an attempt to exclude dust particles which might act as nucleating agents for crystal growth.

The protocol used is shown in Fig. $1 .{ }^{45} \mathrm{Ca}$ $\left({ }^{45} \mathrm{CaCl}_{2} 2 \mathrm{mCi} / \mathrm{mmol}\right.$, Radiochemical Centre, Amersham) was used to monitor crystal formation and was added to the buffer to give approximately $1 \mathrm{nCi}$ / $\mathrm{ml}$ in the final incubation mixture.

Incubations were started by the addition of 0.2 $\mathrm{ml}$ of $\mathrm{Na}_{4} \mathrm{P}_{2} \mathrm{O}_{7} .10 \mathrm{H}_{2} \mathrm{O}$ solution (or $\mathrm{H}_{2} \mathrm{O}$ in controls) to $9 \cdot 8 \mathrm{ml}$ of the buffer containing ${ }^{45} \mathrm{Ca}$. Disposable plastic counting vials were thoroughly rinsed with distilled water and used for incubations to avoid adsorption of PPi to the vessel walls as can happen in glassware.

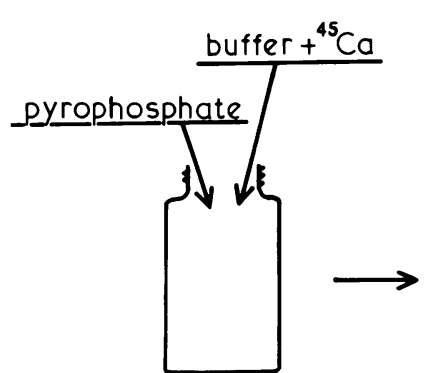

plastic vials
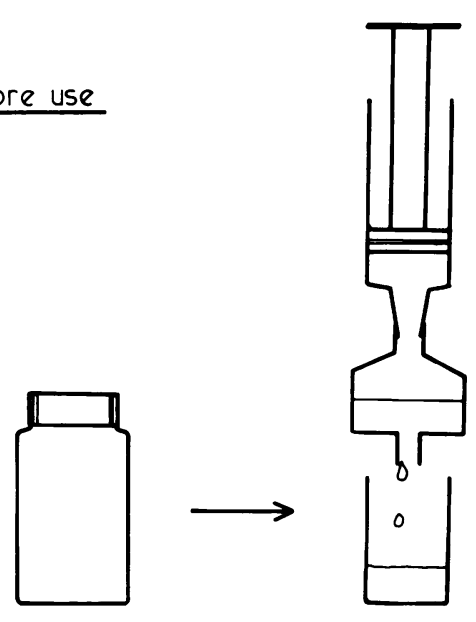

millipore filtration
Fig. 1 Schematic representation of procedure followed for 3-day crystal growth experiments. 
After incubation at $37^{\circ} \mathrm{C}$ for the required time the solutions were filtered through $0.45 \mu \mathrm{m}$ pore-sized Millipore filters to remove any crystals formed. Filters were blotted dry on their underside and placed in scintillation vials while still damp. This avoided loss of crystals from the filter surface which was experienced with dry filters. Once dry the filters were dissolved in a toluene-based scintillant $(600 \mathrm{ml}$ toluene and $400 \mathrm{ml}$ 2-ethoxyethanol $+7 \mathrm{~g}$ PPO) and counted with a Beckman scintillation counter (model LS 7000).

Results are expressed as counts retained by test filters minus counts retained by control filters (the latter having $\mathrm{H}_{2} \mathrm{O}$ in place of $\mathrm{PPi}$ in the incubation). Crystal growth was assumed to have occurred when the test filter held more than 3 times the amount of ${ }^{45} \mathrm{Ca}$ held by control filters. Crystals grown in the presence of buffer $+\mathrm{Ca}^{2+}$ and buffer plus $\mathrm{Mg}^{2+}$ where characterised by $x$-ray diffraction powder patterns (kindly performed by $\mathrm{Dr}$ J. C. Elliot, London Hospital Medical School). Studies were performed with incubations lasting several months and the crystal forms produced were tentatively identified by polarised light microscopy.

\section{Results}

It was found that the quantity of ${ }^{45} \mathrm{Ca}$ incorporated into the crystal phase rose sharply between $12 \mathrm{~h}$ and $48 \mathrm{~h}$. For this reason a standard incubation time of 3 days was used, by which time ${ }^{45} \mathrm{Ca}$ incorporation had levelled off. Only 5 to $10 \%$ additional ${ }^{45} \mathrm{Ca}$ could be incorporated by prolonging the incubation time to 7 days. Various factors were identified which influenced the minimum concentrations of PPi required to promote crystallisation in the presence of $1.5 \mathrm{mmol} / \mathrm{l}$ of calcium.

Effect of $p H$. Fig. 2 shows the effect of $\mathrm{pH}$ on the initiation of crystal growth. The quantity of crystals formed at different concentrations of PPi are shown. It can be seen that higher concentrations of PPi are required to initiate crystal growth under acid conditions; even at $60 \mu$ moles/ $1 \mathrm{PPi}$ there is very little growth at $\mathrm{pH} 6 \cdot 7$. Significantly there is a sharp drop in the concentration of PPi required at around pH $7 \cdot 4$.

At $\mathrm{pH} 7 \cdot 4$ the PPi concentration required for crystallisation was 40 to $45 \mu \mathrm{mol}$ in the presence of $1.5 \mathrm{mmol}$ calcium.

Effect of magnesium. Fig. 3 shows the extent of crystal formation achieved as PPi concentrations were increased in the presence of calcium alone and when magnesium was added at 2 concentrations which represent the limit of the normal range likely

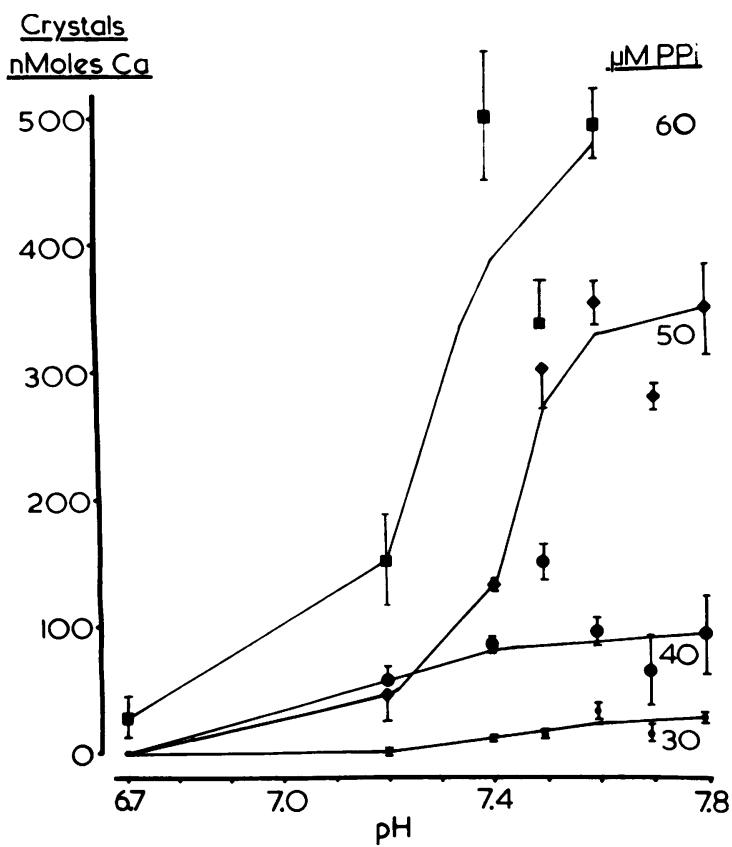

Fig. 2 The quantity of crystals formed (nmol Ca; mean $\pm S E M)$ per vial is plotted against $\mathrm{pH}$ for 4 concentrations of pyrophosphate.

-30 umol ll, $\bullet=50 \mu \mathrm{mol} / \mathrm{l}$, $-40 \mu \mathrm{mol} / \mathrm{l}$, $\square=60 \mu \mathrm{mol} / \mathrm{l}$

Solutions as in Table 1, no magnesium present, 3-day incubations at $37^{\circ} \mathrm{C}$. See text for details.

to be encountered under physiological conditions. In the presence of $0.5 \mathrm{mmol} / 1 \mathrm{Mg}, 175 \mu \mathrm{mol} / 1$ PPi was required; in the presence of $1 \mathrm{mmol} / 1$ $\mathrm{MgCl}_{2}$ the concentration of $\mathrm{PPi}$ required rose to $240 \mu \mathrm{mol} / \mathrm{l}$.

The actual quantity of crystals formed at the highest concentration of PPi tested, as judged by ${ }^{45} \mathrm{Ca}$ retention on filters, was similar in the presence of $\mathrm{Mg}$ as with $\mathrm{Ca}$ alone.

Crystal identification. The crystals grown at $\mathrm{pH}$ 7.4 were shown by $x$-ray diffraction to be the orthorhombic form of $\mathrm{Ca}_{2} \mathrm{P}_{2} \mathrm{O}_{7} \cdot 2 \mathrm{H}_{2} \mathrm{O}$ with an unidentified contaminant. In the presence of $\mathrm{Mg}$ an amorphous phase only was seen after 3-day incubations.

\section{Discussion}

The formation of CaPPD crystals under pathological conditions in vivo could be brought about in several ways. Firstly, the concentrations of either of the two major ions ( $\mathrm{Ca}$ and PPi) might be raised. 
Crystals

nMoles Ca

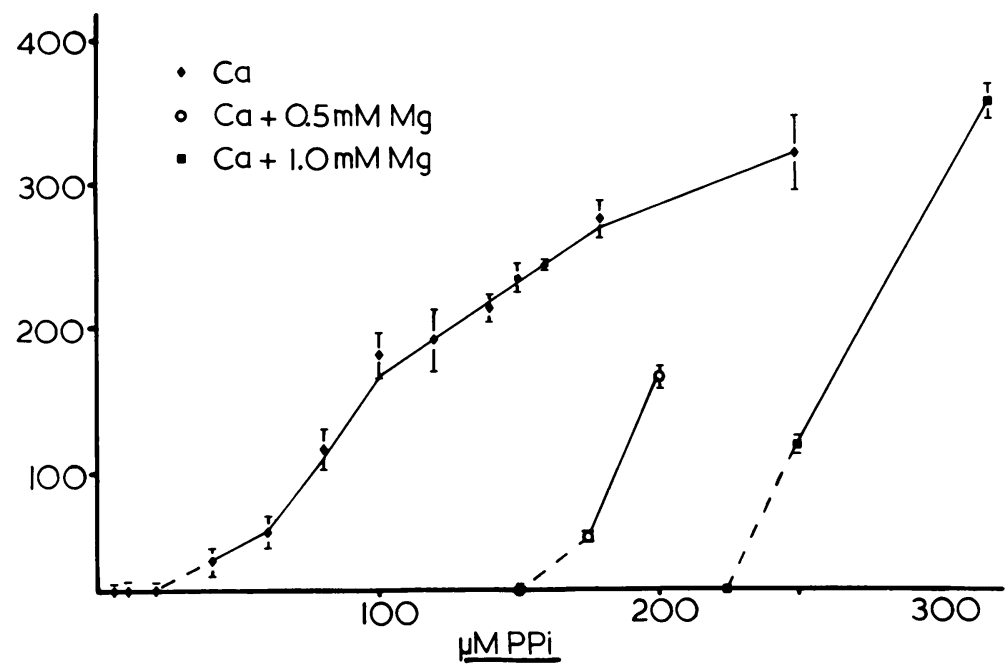

Fig. 3 The effect of $\mathrm{MgCl}_{2}$ on crystal growth (nmol Ca per vial) in 3-day incubations at $37^{\circ} \mathrm{C}$

Raised concentration of the PPi could result from either enhanced production or decreased removal of PPi from the joint. In hypophosphatasia, for example, in which alkaline phosphatase is deficient, body fluid concentrations of PPi are raised, presumably because of defective enzymatic removal. This condition is associated with chondrocalcinosis (Hamilton, 1976). A rise in $\mathrm{Ca}$ concentrations occurs, for example, in hyperparathyroidism which is also known to be associated with CaPPD deposition. Crystal formation might also be favoured by any factor which enhanced their nucleation or diminished the natural concentration of any inhibitors of crystal formation.

The present experiments have defined the concentration of $\mathrm{Ca}$ and $\mathrm{PPi}$ required for crystal growth in the absence of nucleating agents. The concentration of PPi required to form CaPPD crystals at $\mathrm{pH} 7.4$ at physiological ionic strength was around $40 \mu \mathrm{mol} / 1$. This is more than 10 -fold higher than the PPi concentration found in control synovial fluids (mean $3 \mu \mathrm{mol} / 1$, range $1-4$ $\mu \mathrm{mol} / \mathrm{l})$ and double that found in pseudogout fluids (mean $20 \mu \mathrm{mol} / \mathrm{l}$, range 5-60 $\mu \mathrm{mol} / \mathrm{l}$ ). The subsequent addition of $\mathrm{Mg}^{2+}$ at physiological concentrations further raised the concentration of $\mathrm{PPi}$ required to between 175 and $200 \mu \mathrm{mol} / 1$ PPi before crystal formation occurred (Fig. 3). These levels are above even the extremes of PPi recorded for pseudogout fluids and support previous suggestions (McCarty, 1976) that CaPPD crystals are most probably formed within articular cartilage and released into the synovial fluid rather than being formed there de novo. The higher concentration of PPi present in fluids of patients with osteoarthritis and pseudogout may therefore reflect enhanced production and release of PPi from cartilage in these patients.

The concentrations of $\mathrm{Ca}$ and PPi present in articular cartilage are at present unknown but Pita et al. (1978) has reported that pyrophosphatase activity is lower in cartilage from patients with OA or CaPDD than in controls. The high negative fixed charge density (Maroudas, 1968) which exists in articular cartilage may favour retention of cations, and Maroudas (1976) has some evidence which suggests that in this respect cartilage may show some selectivity for calcium. This could lead to a rise in total calcium concentration within cartilage. In normal cartilage the free ionic calcium concentration should remain similar to that in synovial fluid, but one can speculate that under abnormal conditions bound calcium might be released during degradation of proteoglycans and could contribute to the formation of CaPPD crystals.

The effect of pH shown in Fig. 1 is interesting, suggesting that the chances of forming crystals increases rapidly as the $\mathrm{pH}$ rises through the range of $7 \cdot 2-7 \cdot 4$. This effect may be of considerable importance in vivo where a small change in $\mathrm{pH}$ could trigger crystal formation without the need for such large changes in concentrations of $\mathrm{Ca}$ or PPi. The results are reminiscent of those of Bennett et al. (1975), who showed that CaPPD crystals are 


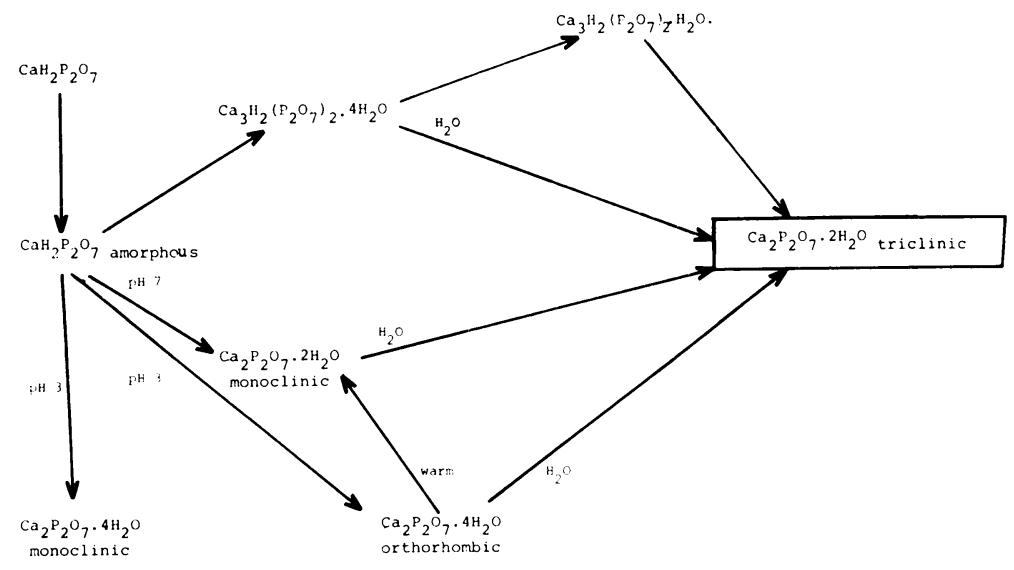

Fig. 4 These interconversions are shown from data given by Lehr and Brown (1967). Most changes in form require $\mathrm{pH}$ or concentration changes, and it is intended only to show that these changes are possible and to infer that crystal interconversions may take place in vivo

more soluble in slightly acidic media. It is of interest to note that extracellular fluid aspirates obtained from epiphyseal cartilage by micropuncture techniques have a high $\mathrm{pH}$ (Cuervo et al., 1971).

The type of CaPPD crystals found in vivo are predominantly the triclinic form (McCarty, 1976), while Bywaters et al. (1978) have recently reported the occurrence of the monoclinic dihydrate crystals, confirming the observations of McCarty et al. (1962). The crystals produced under our experimental conditions were orthorhombic in the absence of $\mathrm{Mg}^{2+}$ and amorphous in the presence of physiological concentrations of $\mathrm{Mg}^{2+}$. These experiments were, however, of only 3 days' duration. Longer incubations of a month or more in the presence of both calcium and magnesium allow the slow formation of crystals of the monoclinic type, followed by slow transition to the triclinic form. These identifications are tentative since they are based on polarised light microscopy. Work is in progress to define those transitions by means of the more definitive $x$-ray diffraction techniques.

It may be of significance that the triclinic crystals are not the first to form in vitro, and the finding of monoclinic crystals in vivo suggests that the most commonly found crystal form, the triclinic CaPPD, is merely the final transition state of different crystal forms. From existing knowledge of the chemistry of calcium pyrophosphate crystals (Lehr and Brown, 1967), as shown in Fig. 4, the triclinic CaPPD does indeed appear to be the final product of almost all crystal-form conversions. An alternative explanation is that the occurrence of monoclinic CaPPD is a manifestation of a crystal deposition disease of a different type from classical pseudogout.
We are very grateful to the Arthritis and Rheumatism Council for a grant to support this work.

\section{References}

Altman, R. D., Muniz, O., Pita, J. C., and Howell, D. S. (1973). Microanalysis of inorganic pyrophosphate (PPi) in synovial fluid and plasma. Arthritis and Rheumatism, 16, 171-178.

Bennett, R. M., Lehr, J. R., and McCarty, D. J. (1975). Factors affecting solubility of calcium pyrophosphate dihydrate crystals. Journal of Clinical Investigation, 56, 1571-1579.

Bundens, C. W. D., Brighton, L. C. C. T., and Weitzman, L. C. G. (1965). Primary articular cartilage calcification with arthritis (pseudogout syndrome). Journal of Bone and Joint Surgery, 47A, 111-122.

Bywaters, E. G. L., Dykes, E., Pirie, C., and Reuben, R. (1978). Crystal deposits in the discs, ligaments, joints and bursae of the spine. Proceedings of the Symposium Studies in Joint Diseases. London Hospital Medical School.

Camerlain, M., McCarty, D. J., Silcox, D. C., and Jung, A. (1975). Inorganic pyrophosphate pool size and turnover rate in arthritic joints. Journal of Clinical Investigation, 55, 1373-1381.

Cuervo, L. A., Pita, J. C., and Howell, P. S. (1971). Ultramicroanalysis of $\mathrm{pH}, \mathrm{PCO}_{2}$ and carbonic anhydrase at calcifying sites in cartilage. Calcified Tissue Research, 7, 220-231.

Hamilton, E. B. D. (1976). Diseases associated with CaPPD deposition disease. Arthritis and Rheumatism, 19, 353-357.

Lehr, J. R., and Brown, E. H. (1967). Chemical Engineering Bulletin 6, May 1967, pp. 22-23. Tennessee Valley Authority: USA.

Maroudas, A. (1968). Physicochemical properties of cartilage in the light of ion exchange theory. Biophysical Journal, 8, 575.

Maroudas, A. (1976). Transport of solutes through cartilage: permeability to large molecules. Journal of Anatomy, 122, 335-347.

McCarty, D. J., Kohn, N. N., and Faires, J. S. (1962). The significance of calcium pyrophosphate crystals in the 
synovial fluid of arthritis patients. The pseudogout syndrome: 1, Clinical aspects. Annals of Internal Medicine, 56, 711-737.

McCarty, D. J., Solomon, S. D., Warnock, M., and Paloyan, E. (1971). Inorganic pyrophosphate concentrations in the synovial fluid of arthritic patients. Journal of Laboratory and Clinical Medicine, 78, 216-229.

McCarty, D. J. (1976). Calcium pyrophosphate dihydrate crystal deposition 1975. Arthritis and Rheumatism, 19, 275-285.

Pita, J. C., Blanco, L. N., and Howell, D. S. (1978). Determination of total and unbound $\mathrm{Ca}$ in nanoliter samples of cartilage fluid and serum. Analytical Biochemistry, 90, 126-135.

Russell, R. G. G., Bisaz, S., Fleisch, H., Currey, H. L. F., Rubinstein, H. M., Dietz, A. A., Boussina, I., Micheli, A., and Fallet, G. (1970). Inorganic pyrophosphate in plasma, urine and synovial fluid of patients with pyrophosphate arthropathy (chondrocalcinosis or pseudogout). Lancet ${ }^{*}$ 2, 899-902.

Silcox, D. C., and McCarty, D. J. (1974). Elevated inorganic pyrophosphate concentrations in synovial fluids in osteoarthritis and pseudogout. Journal of Laboratory and Clinical Medicine, 83, 518-531. 